\title{
Heterogeneous Protection in Regular and Complete Bi-partite Networks (Work in Progress)
}

\author{
Jasmina Omić, Robert E. Kooij, and Piet Van Mieghem \\ Delft University of Technology, \\ Faculty of Electrical Engineering, Mathematics and Computer Science, \\ P.O Box 5031, 2600 GA Delft, The Netherlands \\ $\{$ P.VanMieghem, J.S.Omic, R.E.Kooij\}@ewi.tudelft.nl \\ Dr. ir. Kooij is also with TNO Information Communication Technology, \\ P.O Box 5050, 2600 GB Delft
}

\begin{abstract}
We examine the influence of heterogeneous curing rates for a $S I S$ model, used for malware spreading on the Internet, information dissemination in unreliable networks, and propagation of failures in networks. The topology structures considered are the regular graph which represents the homogenous network structures and the complete bi-partite graph which represents the hierarchical network structures. We find the threshold in a regular graph with $m$ different curing rates.

Further, we consider a complete bi-partite graph with 2 curing rates and find the threshold for any distribution of curing rates among nodes. In addition, we consider the optimization problem and show that the minimum sum of the curing rates that satisfies the threshold equation is equal to the number of links in the graph. The optimization problem is simplified by assuming fixed curing rates $\delta_{1}, \delta_{2}$ and optimization of the distribution of curing rates among different sets of nodes.
\end{abstract}

Keywords: Virus spread, epidemic threshold, heterogeneous networks.

\section{Introduction}

The Susceptible-Infected-Susceptible $(S I S)$ infection model, which arose in mathematical biology, is often used to model the spread of computer viruses [1, 2], 3], epidemic algorithms for information dissemination in unreliable distributed systems like P2P and ad-hoc networks [4, [5], and propagation of faults and failures in networks like $B G P$ [6].

The SIS model assumes that a node in the network is in one of two states: infected and therefore infectious, or healthy and therefore susceptible to infection. The $S I S$ model usually assumes instantaneous state transitions. Thus, as soon as a node becomes infected, it becomes infectious and likewise, as soon as a node is cured it is susceptible to re-infection. There are many models that consider more aspects like incubation periods, variable infection rate, a curing process that takes a certain amount of time and so on [7, 1, 8]. In epidemiological 
theory, many authors refer to an epidemic threshold $\tau_{c}$, see for instance [7], [9], [1] and [3. If it is assumed that the infection rate along each link is $\beta$ while the curing rate for each node is $\delta$ then the effective spreading rate of the virus can be defined as $\tau=\beta / \delta$. The epidemic threshold can be defined as follows: for effective spreading rates below $\tau_{c}$ the virus contamination in the network dies out - the mean epidemic lifetime is of order $\log n$, while for effective spreading rates above $\tau_{c}$ the virus is prevalent, i.e. a persisting fraction of nodes remains infected with the mean epidemic lifetime [2] of the order $e^{n^{\alpha}}$. In the case of persistence we will refer to the prevailing state as a metastable state or steady state. It was shown in [10] and [2] that $\tau_{c}=1 / \rho(A)$ where $\rho(A)$ denotes the spectral radius of the adjacency matrix $A$ of the graph. Recently, the epidemic threshold formula has also been verified by using the $N$-intertwined model [1], which consists of a pair of interacting continuous Markov chains.

It is the aim of this paper to derive results for the epidemic threshold in the case of heterogeneous curing rates for regular and complete bi-partite graphs. A regular graph is an approximation of the random graph for large $N$ and it represents a significant set of networks used in telecommunications. Further, the complete bi-partite graph represents a hierarchal type of topology, also frequently used in telecommunications. Notice that (core) telecommunication networks often can be modeled as a complete bi-partite topology. For instance, the so-called double-star topology (i.e. $K_{M ; N}$ with $M=2$ ) is quite commonly used because it offers a high level of robustness against link failures. For example, the Amsterdam Internet Exchange 1 one of the largest public Internet exchanges in the world, uses this topology to connect its four locations in Amsterdam to two high-throughput Ethernet switches. Sensor networks are also often designed as complete bi-partite graphs.

The rest of the paper is organized as follows. In Section 2, we present the classical model by Kephart and White which describes the homogenous spread of a virus on regular graphs and the SIS model for the complete bi-partite graph also analyzed in [12. In Section 2.1, we derive and analyze the spread of viruses in regular graphs in case of $m$ curing rates. In Section 2.2, we discuss a specific case of regular graphs with 2 curing rates. In Section 2.3, we consider the spread of viruses on the complete bi-partite graphs with two curing rates. In the following section, we give solution for the optimization problem on complete bi-partite graph in the heterogenous case. We summarize our results in Section 4

\section{Virus Spread on Regular and Bi-partite Graphs}

In order to explain our model of spread for computer viruses with heterogeneous curing rates, it is useful to first discuss the spread of viruses with homogeneous curing rate.

The homogenous model for regular graph is based on a classical result by Kephart and White [1] for SIS models. We consider a connected graph with $N$ nodes, where every node has degree $k$. We denote the number of infected

\footnotetext{
${ }^{1}$ see www.ams-ix.net
} 
nodes in the population at time $t$ by $X(t)$. The probability that a randomly chosen node is infected is $v(t) \equiv X(t) / N$. Now, the rate at which the infection probability changes is due to two processes: susceptible nodes becoming infected and infected nodes being cured. The change in probability $v(t)$ due to the curing of infected nodes is $\delta v(t)$. The rate at which the infection probability $v(t)$ grows is proportional to the probability of a node being susceptible, i.e. $1-v(t)$. For every susceptible node, the rate of infection is the product of the infection rate per node $(\beta)$, the degree of the node $(k)$ and the probability that on a given link the susceptible node connects to an infected node $(v(t))$. Therefore, we obtain the following differential equation describing the time evolution of $v(t)$ :

$$
\frac{d v(t)}{d t}=\beta k v(t)(1-v(t))-\delta v(t)
$$

The solution to Eq. (1) is

$$
v(t)=\frac{v_{0}(1-\rho)}{v_{0}+\left(1-\rho-v_{0}\right) e^{-(\beta k-\delta) t}},
$$

where $v_{0}$ is the initial probability of infected nodes. The steady state solution is

$$
v_{\infty}=\frac{\beta k-\delta}{\beta k}
$$

An epidemic steady state only exist for $v_{\infty}>0$, therefore, the epidemic threshold equals to $\tau_{c}=\frac{1}{k}$. For $k$-regular graphs, the spectral radius of the adjacency matrix [13] is equal to $k$, therefore $\tau_{c}=\frac{1}{k}$ is in line with the result in [10].

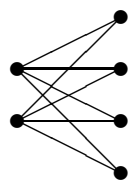

Fig. 1. Complete bi-partite graph $K_{2,4}$

Further, we will consider the complete bi-partite graphs with one curing rate $\delta$. The $S I S$ model for the complete bi-partite graph is presented in [12]. A complete bi-partite graph $K_{M, N}$ consists of two disjoint sets $S_{1}$ and $S_{2}$ containing respectively $M$ and $N$ nodes, such that all nodes in $S_{1}$ are connected to all nodes in $S_{2}$, while within each set no connections occur. Figure 1 gives an example of a complete bi-partite graph with 6 nodes. Since there are two sets of nodes with different degrees, equation (1) does not hold. A node from the set $S_{1}$ is connected to $N$ nodes from the set $S_{2}$. The probability that a randomly chosen node is infected in set $S_{1}$ is $v_{1}(t) \equiv X_{1}(t) / M$. The rate at which probability $v_{1}(t)$ grows is proportional to the probability that a node in the set $S_{1}$ is susceptible multiplied by the degree of the node $N$ and the probability that a node connects to an infected node from set $S_{2}$, which is $v_{2}(t) \equiv X_{2}(t) / N$. For the set $S_{1}$ and $S_{2}$, we can write differential equations 


$$
\begin{aligned}
& \frac{d v_{1}(t)}{d t}=\beta N v_{2}(t)\left(1-v_{1}(t)\right)-\delta v_{1}(t), \\
& \frac{d v_{2}(t)}{d t}=\beta M v_{1}(t)\left(1-v_{2}(t)\right)-\delta v_{2}(t)
\end{aligned}
$$

For $\frac{d v_{1}(t)}{d t}=0$ and $\frac{d v_{2}(t)}{d t}=0$, we have the steady state solution

$$
v_{1 \infty}=\frac{\beta^{2} M N-\delta^{2}}{N \beta(\delta+\beta M)}, v_{2 \infty}=\frac{\beta^{2} M N-\delta^{2}}{M \beta(\delta+\beta N)}
$$

Now, the epidemic threshold equals $\tau_{c}=\left.\frac{\beta}{\delta}\right|_{v_{\infty}=0}=\frac{1}{\sqrt{M N}}$, which is the reciprocal of the spectral radius of the adjacency matrix for the complete bi-partite graph [13].

\subsection{Virus Spread on Regular Graphs with $m$ Curing Rates}

In this section, we derive the threshold for the spread of viruses on regular graphs with $m$ curing rates.

Assume that $n_{1}, n_{2}, . ., n_{m}$ denotes the fraction of nodes with curing rate $\delta_{1}, \delta_{2}, . ., \delta_{m}\left(\sum_{i=1}^{m} n_{i}=1\right)$. It is important to note that one of the assumptions is complete symmetry of the problem. For every node $i$, a fraction $n_{1}$ of neighbors has the curing rate $\delta_{1}$, a fraction $n_{2}$ has curing rate $\delta_{2}$ and so on.

Denote the number of infected nodes of type $i$ in the population at time $t$ by $X_{i}(t)$. The probability that a randomly chosen node of type $i$ is infected is $v_{i}(t) \equiv \frac{X_{i}(t)}{N n_{i}}$. Now, the rate at which the probability of infection for nodes of type $i$ changes is due to two processes: susceptible nodes becoming infected and infected nodes being cured. The curing rate for an infection probability $v_{i}$ is $\delta_{i} v_{i}$. The rate at which the probability $v_{i}$ grows is proportional to the probability of a node of type $i$ being susceptible, i.e. $1-v_{i}$. For every susceptible node the rate of infection is the product of the infection rate per node $(\beta)$ and the probability that on a given link the susceptible node connects to an infected node $\left(\sum_{j=1}^{m}\left(n_{j} k\right) v_{j}\right)$.

Therefore, we obtain the following differential equation describing the time evolution of $v_{i}(t)$ :

$$
\frac{d v_{i}}{d t}=\beta k\left(\sum_{j=1}^{m} n_{j} v_{j}\right)\left(1-v_{i}\right)-\delta_{i} v_{i}, i=1, . ., m
$$

Note that for $\delta_{1}=\delta_{2}=. .=\delta_{m}$, the system of equations (4) reduces to Eq. (11) with $v=\sum_{j=1}^{m} n_{j} v_{j}$.

For the general case with different curing rates, it is impossible to obtain an explicit solution for the system of equations (4). The standard approach for this type of system of nonlinear differential equations, is to study the qualitative behavior in the phase space.

Theorem 1. Consider connected regular graphs where each node has exactly $k$ neighbors. Assume that the infection rate along each link is $\beta$ while the curing 
rate for each node is $\delta_{i}$ for a fraction $n_{i}$ of the nodes, with $i=1, . ., m \leqslant k$ and $\sum_{i=1}^{m} n_{i}=1$. Complete symmetry is assumed, where each node sees the same fraction of different curing rates. If we define the effective spreading rate as $\tau=\frac{\beta}{\delta^{*}}$, where $\delta^{*}$ is defined as the weighted harmonic mean of $\delta_{1}, \ldots, \delta_{m}$, i.e. $\delta^{*}=\left(\sum_{i=1}^{m} \frac{n_{i}}{\delta_{i}}\right)^{-1}$, then the epidemic threshold satisfies $\tau_{c}=\frac{1}{k}$.

Proof. We denote the fraction of infected nodes of type $i(1 \leqslant i \leqslant m)$ at time $t$ as $v_{i}(t)$. This leads to a system of $m$ differential equations (4).

We will use a Lyapunov function [14 to show that, under the condition $\beta \sum_{t=1}^{m} \frac{n_{t}}{\delta_{t}}-\frac{1}{k} \leq 0$, the origin is a global attractor for $\left\{v_{1} \geq 0, v_{2} \geq 0, . ., v_{m} \geq 0\right\}$, hence, that the virus dies out. Let $V\left(v_{1}, v_{2}, . ., v_{m}\right)=\prod_{j=1}^{m} \delta_{j} \sum_{s=1}^{m} \frac{v_{s}}{\delta_{s}}$. Then, we have

$$
\begin{aligned}
\frac{d V}{d t} & =-\left(\sum_{s=1}^{m} v_{s}\right)\left(\beta k V-\beta k \prod_{j=1}^{m} \delta_{j} \sum_{t=1}^{m} \frac{n_{t}}{\delta_{t}}+\prod_{j=1}^{m} \delta_{j}\right) \\
& =-\left(\sum_{s=1}^{m} v_{s}\right)\left(\beta k V-k \prod_{j=1}^{m} \delta_{j}\left(\beta \sum_{t=1}^{m} \frac{n_{t}}{\delta_{t}}-\frac{1}{k}\right)\right) .
\end{aligned}
$$

The claim follows directly by applying Lyapunov's stability theorem. Next we consider the case $\beta \sum_{t=1}^{m} \frac{n_{t}}{\delta_{t}}-\frac{1}{k}>0$. We first note that any trajectory of the system (44) can never leave the box $B=\left\{\left(v_{1}, \ldots, v_{m}\right) \mid 0 \leq v_{1} \leq 1, \ldots, 0 \leq v_{m} \leq 1\right\}$. This follows from $\left.\frac{d v_{1}}{d t}\right|_{v_{1}=0}=\beta k\left(\sum_{j=1}^{m} n_{j} v_{j}\right) \geq 0$, and similar inequalities at the borders of the box $B$.

From the construction of the above Lyapunov function $V$, we can see that for $\beta \sum_{t=1}^{m} \frac{n_{t}}{\delta_{t}}-\frac{1}{k}>0$, and for $\left(v_{1}, \ldots, v_{m}\right) \in B$ and sufficiently close to the origin, $\frac{d V}{d t}>0$. This implies that the origin has an unstable manifold in $B$. Therefore, since any trajectory of system (4) can never leave the box $B$, system (4) has an attractor as the $\omega$-limit set and, hence, the virus does survive. This finishes the proof of the theorem.

\subsection{Virus Spread on Regular Graphs with Two Curing Rates}

The two dimensional case $(m=2)$ of virus spread on a regular graph can be analyzed in more details. Applying Theorem 1. the spreading process has a threshold at $\tau=\frac{\beta}{\delta^{*}}=\frac{1}{k}$, where $\delta^{*}=\frac{\delta_{1} \delta_{2}}{n_{1} \delta_{2}+n_{2} \delta_{1}}$. 


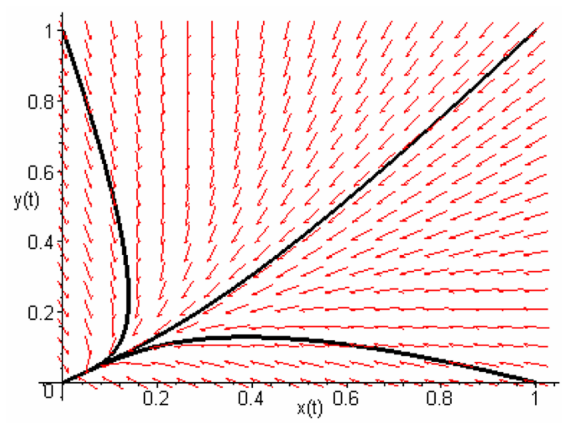

a)

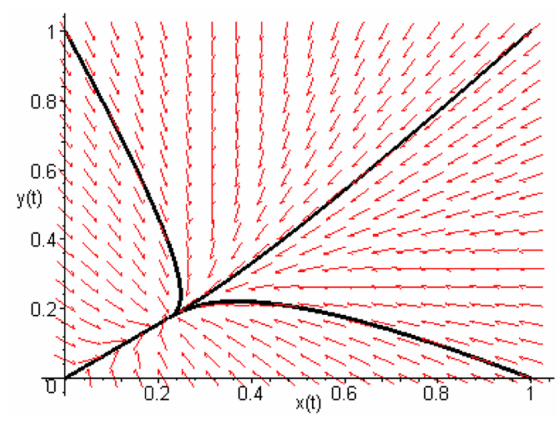

b)

Fig. 2. Phase portrait for a regular graph with the two curing rates where a) virus dies out $\beta=0.2, \delta_{1}=0.8, \delta_{2}=1.2, k=4, n_{1}=n_{2}=0.5$. b) virus survives $\beta=0.4$, $\delta_{1}=0.8, \delta_{2}=1.2, k=4, n_{1}=n_{2}=0.5$.

The phase portrait of two examples are depicted in Figure 2 The attractor for the case where virus survives is given by $\left(v_{1}, v_{2}\right)=(0.22,0.17)$.

For system (4) where $m=2$, it can be proven that the attractor is an equilibrium point of a nodal type, situated on a straight line $L$. It can also be shown that the system does not contain other equilibrium points in $A$ or closed orbits. Therefore, in the case $m=2$, this equilibrium point is a global attractor of system (4) in $A$.

Lemma 1. The set of differential equations given by (4) for $m=2$, has a straight line solution of the form $v_{2}=\lambda v_{1}$.

Proof. We have that

$$
\begin{gathered}
\left(\frac{d v_{2}}{d t}=\lambda \frac{d v_{1}}{d t}\right)_{v_{2}=\lambda v_{1}} \\
-v_{1}\left(\beta k n_{1} \lambda^{2}+\left(\beta k\left(n_{1}-n_{2}\right)-\delta_{1}+\delta_{2}\right) \lambda-\beta k n_{2}\right) \equiv-v_{1} f(\lambda)
\end{gathered}
$$

$f(\lambda)$ has got exactly one negative root and one positive root. The positive root $\lambda_{1}$ satisfies

$$
\lambda_{1}=\frac{\beta k\left(n_{2}-n_{1}\right)+\delta_{1}-\delta_{2}+\sqrt{\Delta}}{2 \beta k n_{1}},
$$

where $\Delta=\beta^{2} k^{2}+2 \beta k\left(n_{1}-n_{2}\right)\left(\delta_{2}-\delta_{1}\right)+\left(\delta_{1}-\delta_{2}\right)^{2}$. Therefore the straight line $L: v_{2}=\lambda_{1} v_{1}$ is a solution of system (4) for $m=2$, which for $0 \leq v_{1} \leq 1$ is situated in $A$.

By application of the Poincaré-Bendixson theorem [14] on $A$, the $\omega$-limit set for the system (4) for $m=2$, can be either an equilibrium point or an isolated periodic orbit. From the fact that there is a line solution through the equilibrium point, it follows that the $\omega$-limit set is the equilibrium point. 


\subsection{Virus Spread on Complete Bi-partite Graphs with Two Curing Rates}

We will now derive a model for virus spread on the complete bi-partite graph $K_{M, N}$ with two different spreading rates. The result is general and it can be reduced to the case with all nodes in one set $\left(S_{1}\right)$ having one curing rate $\delta_{1}$ and in the other $\left(S_{2}\right) \delta_{2}$.

Let us assume that a fraction $p$, with $p \in[0,1]$, of nodes belonging to $S_{1}$ and a fraction $q$, with $q \in[0,1]$, of nodes belonging to set $S_{2}$ have a curing rate $\delta_{1}$, the rest have a curing rate $\delta_{2}$. The total fraction of nodes with the curing rate $\delta_{1}$ is $s=\frac{M p+N q}{M+N}$.

Denote the number of infected nodes of type 1 in the population of nodes from set $S_{1}$ at time $t$ by $X_{i 1}(t)$. The probability that a randomly chosen node of type 1 from set $S_{1}$ is infected is $v_{i 1}(t) \equiv \frac{X i_{1}(t)}{M p}$. Similarly, let $v_{i 2}$ denote the infection probability for nodes of type 2 from set $S_{1},\left(v_{j 1}\right.$ denotes type 1 , set $S_{2}$; and $v_{j 2}$ denotes type 2 , set $S_{2}$ ). Now, the rate at which the probability of infection for nodes of type 1 , set $S_{1}$ changes is due to two processes: susceptible nodes becoming infected and infected nodes being cured. The curing rate for an infection probability $v_{i 1}$ for nodes of type 1 , set $S_{1}$ is $\delta_{1} v_{i 1}$. The rate at which the probability $v_{i 1}$ grows is proportional to the probability of a node of type 1 , set $S_{1}$ being susceptible, i.e. $1-v_{i 1}$. For every susceptible node the rate of infection is the product of the infection rate per node $(\beta)$, the degree of the node $(N)$ and the probability that on a given link the susceptible node connects to an infected node $\left(q v_{j 1}+(1-q) v_{j 2}\right)$.

Similarly, we obtain the differential equations for the other probabilities $\left(v_{i 2}, v_{j 1}, v_{j 2}\right)$ :

$$
\left\{\begin{array}{l}
\frac{d v_{i 1}}{d t}=\beta N\left(q v_{j 1}+(1-q) v_{j 2}\right)\left(1-v_{i 1}\right)-\delta_{1} v_{i 1}, \\
\frac{d v_{i 2}}{d t}=\beta N\left(q v_{j 1}+(1-q) v_{j 2}\right)\left(1-v_{i 2}\right)-\delta_{2} v_{i 2}, \\
\frac{d v_{j 1}}{d t}=\beta M\left(p v_{i 1}+(1-p) v_{i 2}\right)\left(1-v_{j 1}\right)-\delta_{1} v_{j 1}, \\
\frac{d v_{j 2}}{d t}=\beta N\left(p v_{i 1}+(1-p) v_{i 2}\right)\left(1-v_{j 2}\right)-\delta_{2} v_{j 2},
\end{array}\right.
$$

The same set of equations can be obtained by the $N$-intertwined model [1].

In order to simplify the system of equations, we will substitute

$$
i_{1}=p v_{i 1}, i_{2}=(1-p) v_{i 2}, j_{1}=q v_{j 1}, j_{2}=(1-q) v_{j 2}
$$

and

$$
i=i_{1}+i_{2}, j=j_{1}+j_{2}
$$

Therefore, we obtain the following differential equations for $i_{1}(t), i_{2}(t), j_{1}(t)$, $j_{2}(t)$ :

$$
\left\{\begin{array}{l}
\frac{d i_{1}}{d t}=p \beta N j-\beta N j i_{1}-\delta_{1} i_{1}, \\
\frac{d i_{2}}{d t}=(1-p) \beta N j-\beta N j i_{2}-\delta_{2} i_{2}, \\
\frac{d j_{1}}{d t}=q \beta M i-\beta M i j_{1}-\delta_{1} j_{1}, \\
\frac{d j_{2}}{d t}=(1-q) \beta N i-\beta N i j_{2}-\delta_{2} j_{2},
\end{array}\right.
$$


By solving the system of equations 6 for the steady state $\left(\frac{d i_{1}}{d t}=\frac{d i_{2}}{d t}=\frac{d j_{1}}{d t}=\right.$ $\left.\frac{d j_{2}}{d t}=0\right)$ we can calculate the threshold:

$$
\begin{gathered}
\frac{\beta}{\delta^{*}}=\tau_{c}=\frac{1}{\sqrt{M N}} \\
\delta^{*}=\frac{\delta_{1} \delta_{2}}{\sqrt{\delta_{1}^{2}(1-p)(1-q)+\delta_{2}^{2} p q+\delta_{1} \delta_{2}(p(1-q)+q(1-p))}}
\end{gathered}
$$

Theorem 2. Consider complete bi-partite graphs $K_{M, N}$ consisting of two disjoint sets $S_{1}$ and $S_{2}$ containing respectively $M$ and $N$ nodes. Assume that the infection rate along each link is $\beta$. For the nodes in $S_{1}$ a fraction $p$ has curing rate $\delta_{1}$ and in $S_{2}$ a fraction $q$ of the nodes has curing rate $\delta_{1}$, while the curing rate for a fraction $(1-p)((1-q))$ of the nodes is $\delta_{2}$. If we define the effective spreading rate as $\tau=\frac{\beta}{\delta^{*}}$, where $\delta^{*}$ is defined as $\delta^{*}=\frac{\delta_{1} \delta_{2}}{\sqrt{(1-p)(1-q) \delta_{1}^{2}+p q \delta_{2}^{2}+\delta_{1} \delta_{2}(p(1-q)+q(1-p))}}$, then the epidemic threshold satisfies $\tau_{c}=\frac{1}{\sqrt{M N}}$.

Proof. First, we will show that if $\frac{\beta}{\delta^{*}} \leqslant \frac{1}{\sqrt{M N}}$, the virus dies out. $(0,0,0,0)$ is an equilibrium point for system (5). We will use a Lyapunov function to show that, under the condition $\frac{\beta}{\delta^{*}} \leqslant \frac{1}{\sqrt{M N}}$, the origin is a global attractor for $i_{1} \geq 0, i_{2} \geq 0, j_{1} \geq 0, j_{2} \geq 0$.

Let $V\left(i_{1}, i_{2}, j_{1}, j_{2}\right)=\delta_{1} \delta_{2}^{2} i_{1}+\delta_{1}^{2} \delta_{2} i_{2}+\beta N\left(p \delta_{2}+(1-p) \delta_{1}\right)\left(\delta_{2} j_{1}+\delta_{1} j_{2}\right)$. Then,

$$
\begin{aligned}
\frac{d V}{d t} & =\left(\beta ^ { 2 } M N \left((1-p)(1-q) \delta_{1}^{2}+p q \delta_{2}^{2}+\right.\right. \\
& \left.\left.+\delta_{1} \delta_{2}((1-p) q+(1-q) p)\right)-\delta_{1}^{2} \delta_{2}^{2}\right)\left(i_{1}+i_{2}\right) \\
& -\beta N \delta_{2}\left(\beta M\left(p \delta_{2}+(1-p) \delta_{1}\right)+\delta_{1} \delta_{2}\right) i_{1} j_{1} \\
& -\beta N \delta_{1}\left(\beta M\left(p \delta_{2}+(1-p) \delta_{1}\right)+\delta_{2}^{2}\right) i_{1} j_{2} \\
& -\beta N \delta_{2}\left(\beta M\left(p \delta_{2}+(1-p) \delta_{1}\right)+\delta_{1}^{2}\right) i_{2} j_{1} \\
& -\beta N \delta_{1}\left(\beta M\left(p \delta_{2}+(1-p) \delta_{1}\right)+\delta_{1} \delta_{2}\right) i_{2} j_{2} .
\end{aligned}
$$

The extinction of the virus follows directly by applying Lyapunov's stability theorem. Next we will show that if $\frac{\beta}{\delta^{*}}>\frac{1}{\sqrt{M N}}$, the virus survives. We first note that any trajectory of the system (5) can never leave the box $B=\left\{\left(i_{1}, i_{2}, j_{1}, j_{2}\right) \mid 0 \leq\right.$ $\left.i_{1} \leq 1,0 \leq i_{2} \leq 1,0 \leq j_{1} \leq 1,0 \leq j_{2} \leq 1\right\}$. This follows from $\left.\frac{d i_{1}}{d t}\right|_{i_{1}=0}=$ $p \beta N\left(j_{1}+j_{2}\right) \geq 0$, and similar inequalities at the borders of the box $B$.

From the construction of the Lyapunov function, we can observe that for $\left.\beta^{2} M N\left((1-p)(1-q) \delta_{1}^{2}+p q \delta_{2}^{2}+\delta_{1} \delta_{2}((1-p) q+(1-q) p)\right)-\delta_{1}^{2} \delta_{2}^{2}\right)-\delta_{1}^{2} \delta_{2}^{2}>0$ and for $\left(i_{1}, i_{2}, j_{1}, j_{2}\right) \in B$ and sufficiently close to the origin, $\frac{d V}{d t}>0$. This implies that the origin has an unstable manifold in $B$. Therefore, because any trajectory of system (5) can never leave the box $B$, system (5) has an attractor as the $\omega$-limit set and hence the virus does survive.

The result from Theorem 2 holds for non-symmetric cases: a node from set $S_{1}$ sees different portion of nodes with curing rate $\delta_{1}$ than a node from set $S_{2}$ 
$(p \neq q)$. In the symmetric case $(p=q)$, a more general result with $m$ different curing rates can be derived, as in the case of the regular graph, described in Theorem 1 .

\section{Optimal Heterogenous Protection of Complete Bi-partite Graphs}

We will not consider the simple case of optimization for a regular graph.

For any bi-partite graph, the threshold for the heterogenous case is fixed and equal to $\delta^{*}=\beta \sqrt{M N}$. The threshold can be reached for different values of $\delta_{1}, \delta_{2}, p$ and $q$. For example, for $\left(\delta_{1}=\beta M, \delta_{2}=\beta N, p=1, q=0\right)$ the threshold is reached with $\delta_{1}$ applied on nodes from set $S_{1}$, while for $\left(\delta_{1}=\beta M, \delta_{2}=\beta N, p=\right.$ $1, q=0)$ the threshold is also reached and the curing rate $\delta_{1}$ is now applied on the nodes from the other set. The question is how can we decide which solution is better. One of the options is to minimize the total protection strategy applied on the network, while reaching the threshold. The total protection strategy can be defined as a sum of all protection strategies and we will denote it by $D$

$$
D=\sum_{l=1}^{M+N} \delta_{l}=M p \delta_{1}+M(1-p) \delta_{2}+N q \delta_{1}+N(1-q) \delta_{2}
$$

For the previous two cases, the total protection strategy is different. In case $\left(\delta_{1}=\right.$ $\left.\beta M, \delta_{2}=\beta N, p=1, q=0\right)$, the total protection strategy is $D=\beta\left(M^{2}+N^{2}\right)$, and in the other case, $D=2 \beta M N$, which is always smaller than or equal to the first case.

Let us formulate the optimization problem as follows:

Problem 1. Minimize

$$
D=M p \delta_{1}+M(1-p) \delta_{2}+N q \delta_{1}+N(1-q) \delta_{2}
$$

subject to the conditions

$$
\begin{gathered}
\beta \sqrt{M N}=\frac{\delta_{1} \delta_{2}}{\sqrt{(1-p)(1-q) \delta_{1}^{2}+p q \delta_{2}^{2}+\delta_{1} \delta_{2}(p(1-q)+q(1-p))}} \\
0 \leq p, q \leq 1 \\
0<\delta_{1}, \delta_{2}
\end{gathered}
$$

The optimization problem is non-linear with non-linear conditions. However, from [15, we know that the minimum of the function $D$ for any graph and any set of curing rates is equal to the number of links $L$ in the network, multiplied by $2 \beta$,

$$
D_{\min }=2 \beta L \text {. }
$$

In the case of the complete bi-partite graph, the minimum is $D_{\min }=2 \beta M N$ and it is reached for $\left(\delta_{1}=\beta M, \delta_{2}=\beta N, p=1, q=0\right)$ or $\left(\delta_{1}=\beta N, \delta_{2}=\beta M, p=\right.$ $0, q=1)$. This means that for $N>M$, the larger curing rate proportional to the number of links in set $S_{1}$ will be assigned to the nodes from that set. The larger curing rate is assigned to the more connected nodes. 
Further, we can have a situation, where curing rates $\left(\delta_{1}, \delta_{2}\right)$ are fixed and we will optimize the parameters $(p, q)$. This optimization problem can be formulated as follows.

Problem 2. For two fixed curing rates $\delta_{1}, \delta_{2}$, minimize function (8), subject to the conditions (10).

From the threshold condition we can determine one of the variables $p$ or $q$. We will derive equations for variable $q$ (the case with $p$ is analogue),

$$
q=\frac{\delta_{1}\left(M N \delta_{1}(1-p)+M N \delta_{2} p-\delta_{1} \delta_{2}^{2}\right)}{M N\left(\delta_{1}^{2}(1-p)+\delta_{1} \delta_{2}(2 p-1)+\delta_{1}^{2}\right)}
$$

By substituting $q$ in $D$, the total sum of curing rates becomes a function of parameter $p$ only and optimization is simplified. The function is of the form $D(p)=\frac{P_{2}(p)}{P_{1}(p)}$ where $P_{1}(p)$ is a polynomial of the first order in $p$ and $P_{2}(p)$ is a polynomial in the second order in $p$.

Lemma 2. For any fixed $\delta_{1}, \delta_{2}$, the optimal solution of minimization problem 2 is on the boundary of the region $(p=0$ or $p=1$ or $q=0$ or $q=1)$.

Proof. The function $D(p)$ is not defined for $P_{1}(p)=0$, which holds for $p=\frac{\delta_{1}}{\delta_{1}-\delta_{2}}$. The value $\frac{\delta_{1}}{\delta_{1}-\delta_{2}}$ does not belong to the interval $[0,1]$. The second derivative of $D(p)$ is strictly negative in the interval $q \in[0,1]$.

$$
\frac{d^{2} D(p)}{d p^{2}}=-\frac{2 \delta_{1}^{2} \delta_{2}^{2}\left(\delta_{1}-\delta_{2}\right)^{2}}{\left(\delta_{1}(1-q)+\delta_{2} q\right)}<0, q \in[0,1]
$$

Therefore, $D(p)$ is concave in the interval of interest and minimum is on the boundaries of the interval.

For given $\delta_{1}, \delta_{2}$, it is not always possible to reach the threshold. In the case $\delta_{1}, \delta_{2}<\beta \sqrt{M N}$, the threshold cannot be reached and the network is in the state of permanent infection. For example, if $\delta_{1}, \delta_{2}<\beta \sqrt{M N}$ and $\delta_{1}>\delta_{2}$, if we take only the larger curing rate for the whole network, we have $\frac{\beta}{\delta_{1}}<\beta \frac{1}{\sqrt{M N}}$. In the case $\delta_{1}, \delta_{2}>\beta \sqrt{M N}$, the network is cured, however, the network is above the threshold and a higher curing rate than necessary is applied.

If the threshold can be reached, Lemma 2 shows that either set $S_{1}$ or set $S_{2}$ is completely protected with only one curing rate. In order to minimize the sum of curing rates we are interested how many times we can apply smaller curing rates. Without loss of generality, let $\delta_{1}<\beta \sqrt{M N}<\delta_{2}$ and $N>M$. Firstly, we will assign $\delta_{2}$ to all the nodes from larger set with $N$ nodes and $\delta_{1}$ to the smaller set. If the effective spreading rate obeys $\frac{\beta}{\delta^{*}}>\frac{1}{\sqrt{M N}}$, than $p=1$, and $q$ can be calculated from equation (11). In the case $\frac{\beta}{\delta^{*}}<\frac{1}{\sqrt{M N}}$, the network is cured and below the threshold. Then $q=0$ and $p$ can be calculated from the condition for the threshold. 


\section{Conclusion}

The epidemic theory is widely applied on many networking problems. The SIS model, on which we have focused here, is applied in malware modeling in the Internet [1, 2], 3], information dissemination in P2P and ad-hoc networks [4, [5] and propagation of faults and failures [6. The two types of topologies that we considered, namely the regular graph and the complete bi-partite graph, arise as subnet structures in telecommunication networks. We have studied the influence of heterogenous protection in regular and complete bi-partite graphs.

Using Lyapunov's stability theorem, we have shown that for regular graphs, the epidemic threshold satisfies $\frac{\beta}{\delta^{*}}=\frac{1}{k}$, where $\delta^{*}$ is defined as the weighted harmonic mean of $\delta_{1}, \ldots, \delta_{m}$. This result holds under the assumption of complete symmetry, where each node sees the same fraction of different curing rates. Without this assumption, the problem becomes significantly complex [16].

Further, we have considered the heterogenous case with 2 curing rates for the complete bi-partite graph. The threshold, given by Eq. (7) becomes the geometric mean of curing rates $\delta_{1}, \delta_{2}$ for $p=1, q=0$ and the weighted harmonic mean if $p=q$. For other values of $p$ and $q$, total curing rate $\delta^{*}$ belongs to the interval $\left[\delta_{1}, \delta_{2}\right]$.

Many different pairs of curing rate can satisfy the threshold equation, therefore the question which solution is more optimal rises. We consider the optimality of heterogeneous protections for complete bi-partite graph with the respect to sum of all applied curing rates and concluded that global optimum in this respect is equal to the number of links in the complete bi-partite graph. For the case of fixed $\delta_{1}$ and $\delta_{2}$, the optimal solution is on the boundaries of $(p, q) \in[0,1] \times[0,1]$.

\section{References}

1. Kephart, J.O., White, S.R.: Measuring and modeling computer virus prevalence. In: IEEE Symposium on Security and Privacy (1993)

2. Ganesh, A., Massoulié, L., Towsley, D.: The effect of network topology on the spread of epidemics. In: Proceedings of IEEE INFOCOM, pp. 1455-1466 (2005)

3. Pastor-Satorras, R., Vespignani, A.: Epidemic spreading in scale-free networks. Phys. Rev. Lett. 86(14), 3200-3203 (2001)

4. Chakrabarti, D., Leskovec, J., Faloutsos, C., Madden, S., Guestrin, C., Faloutsos, M.: Information survival threshold in sensor and p2p networks. In: Proceedings of IEEE INFOCOM (2007)

5. Eugster, P., Guerraoui, R., Kermarrec, A.M., Massoulie, L.: From epidemics to distributed computing. IEEE Computer 37, 60-67 (2004)

6. Coffman, F.G.J., Ge, Z., Misra, V., Towsley, D.: Network resilience: exploring cascading failures within bgp. In: Proc. 40th Annual Allerton Conference on Communications, Computing and Control (2002)

7. Daley, D.J., Gani, J.: Epidemic modelling: An Introduction. Cambridge University Press, Cambridge (1999)

8. Wang, Y., Wang, C.: Modeling the effects of timing parameters on virus propagation. In: WORM 2003: Proceedings of the 2003 ACM workshop on Rapid malcode, pp. 61-66. ACM, New York (2003) 
9. Bailey, N.T.J.: The Mathematical Theory of Infectious Diseases and its Applications. Charlin Griffin \& Company (1975)

10. Wang, Y., Chakrabarti, D., Wang, C., Faloutsos, C.: Epidemic spreading in real networks: An eigenvalue viewpoint. In: IEEE Symposium on Reliable Distributed Systems. IEEE Computer Society, Los Alamitos (2003)

11. Van Mieghem, P., Omic, J., Kooij, R.: Epidemic spreading in scale-free networks. IEEE Transactions on Networking, 1-14 (2009)

12. Omic, J., Kooij, R., Van Mieghem, P.: Virus spread in complete bi-partite graphs. In: Proceedings of BIONETICS (2007)

13. Cvetkovic, D.M., Doob, M., Sachs, H.: Spectra of graphs, Theory and Applications, 3rd Revised and Enlarged Edition. Johan Ambrosius Barth Verlag (1998)

14. Guckenheimer, J., Holmes, P.: Nonlinear oscillations, dynamical systems, and bifurcations of vector fields. Springer, New York (1983)

15. Omic, J., Orda, A., Van Mieghem, P.: Protecting against network infections: A game theoretic perspective. In: Proceedings of IEEE INFOCOM (2009)

16. Van Mieghem, P., Omic, J.: In-homogeneous virus spread in networks. TUDelft Technical report 20080801 (2008) 